\section{RMD Open}

Rheumatic \&

Musculoskeletal Diseases

\title{
Low rates of radiographic progression of structural joint damage over 2 years of baricitinib treatment in patients with rheumatoid arthritis
}

To cite: van der Heijde D, Schiff M, Tanaka Y, et al. Low rates of radiographic progression of structural joint damage over 2 years of baricitinib treatment in patients with rheumatoid arthritis. RMD Open 2019;5:e000898. doi:10.1136/ rmdopen-2019-000898

\section{- Additional material is} published online only. To view please visit the journal online (http://dx.doi.org/10.1136/ rmdopen-2019-000898).

Received 7 January 2019 Revised 22 April 2019 Accepted 23 April 2019
Check for updates

\section{(C) Author(s) (or their} employer(s)) 2019. Re-use permitted under CC BY-NC. No commercial re-use. See rights and permissions. Published by BMJ.

For numbered affiliations see end of article.

\section{Correspondence to}

Professor Desirée van der Heijde; mail@dvanderheijde.nl

\author{
Desirée van der Heijde, ${ }^{\oplus 1,2}$ Michael Schiff, ${ }^{3}$ Yoshiya Tanaka, ${ }^{\circ}$ Li Xie, $^{5}$ \\ Gabriella Meszaros, ${ }^{5}$ Taeko Ishii, ${ }^{5}$ Marta Casillas, ${ }^{5}$ Robert A Ortmann, ${ }^{5}$ \\ Paul Emery ${ }^{6,7}$
}

\section{ABSTRACT}

Objectives To evaluate radiographic progression of structural joint damage over 2 years in patients with rheumatoid arthritis from baricitinib clinical trials who were disease-modifying antirheumatic drug (DMARD)-naïve or had an inadequate response to conventional synthetic DMARDs (csDMARD-IR).

Methods Patients had completed one of three phase III studies and entered a long-term extension (LTE) study, continuing on the same baricitinib dose as at originating study completion. At 52 weeks, DMARD-naïve patients receiving methotrexate (MTX) or combination therapy (baricitinib $4 \mathrm{mg}+\mathrm{MTX}$ ) were switched to baricitinib $4 \mathrm{mg}$ monotherapy ( \pm MTX per investigator opinion); MTX-IR patients receiving adalimumab were switched to baricitinib $4 \mathrm{mg}$ on background MTX. At 24 weeks, csDMARD-IR patients receiving placebo were switched to baricitinib $4 \mathrm{mg}$ on background csDMARD. Radiographs at baseline, year 1 and year 2 were scored using the van der Heijde modified Total Sharp Score. Linear extrapolation was used for missing data.

Results Of 2573 randomised patients, 2125 (82.6\%) entered the LTE, of whom 1893 (89.1\%) entered this analysis. At year 2, progression was significantly lower with initial baricitinib (monotherapy or combination therapy) versus initial MTX in DMARD-naïve patients (proportion with non-progression defined by ssmallest detectable change (SDC): $87.3 \%$ baricitinib $4 \mathrm{mg}+\mathrm{MTX}$; $70.6 \%$ MTX; $p \leq 0.001$ ). In MTX-IR patients, progression with initial baricitinib was significantly lower than with initial placebo and similar to initial adalimumab (SSDC: $82.7 \%$ baricitinib $4 \mathrm{mg} ; 83.5 \%$ adalimumab; $70.6 \%$ placebo; $p \leq 0.001$ ). In csDMARD-IR patients, significant benefit was seen with baricitinib $4 \mathrm{mg}$ ( $\leq$ SDC: $87.2 \%$ vs $73.2 \%$ placebo; $p \leq 0.01$ ).

Conclusions Treatment with once-daily baricitinib resulted in low rates of radiographic progression for up to 2 years.

\section{INTRODUCTION}

Rheumatoid arthritis (RA) is a chronic, systemic autoimmune disease. Persistent

\section{Key messages}

What is already known about this subject?

- Three phase III clinical studies (RA-BEGIN, RA-BEAM, RA-BUILD) reported significant reductions in radiographic progression in baricitinib treatment groups for up to 1 year of treatment, including in patients who were disease-modifying antirheumatic drug (DMARD)-naïve or had previously failed to adequately improve on conventional synthetic DMARDs (csDMARDs) alone.

What does this study add?

- The current analysis extends previous findings, demonstrating that treatment with baricitinib 2 and $4 \mathrm{mg}$ maintains the inhibition of structural joint damage for up to 2 years in patients who completed one of the three original studies and entered the longterm extension study, RA-BEYOND.

How might this impact on clinical practice?

- These data show that baricitinib, as monotherapy or in combination with csDMARDs, provides sustained structural benefits for up to 2 years in a broad spectrum of patients with rheumatoid arthritis (including DMARD-naïve patients and patients with an inadequate response to methotrexate and other CsDMARDS).

- Both 2 and $4 \mathrm{mg}$ doses of baricitinib provided reduction on structural damage progression, with the most robust evidence seen for the $4 \mathrm{mg}$ dose.

inflammation can lead to structural joint damage, cartilage loss and erosive bone damage, which are associated with disability and poor patient outcomes. ${ }^{12}$ Current recommendations advise early treatment with disease-modifying antirheumatic drugs (DMARDs) to prevent or delay structural progression and the resulting impairments to function. ${ }^{3-5}$ 
Baricitinib is an oral, reversible inhibitor of Janus kinase (JAK) 1 and JAK2, which mediate signal transduction for a variety of cytokines involved in inflammatory conditions, including RA. ${ }^{67}$ Baricitinib is approved for the treatment of moderately to severely active RA in adults in over 55 countries, including European countries, Japan and the USA. Three phase III studies previously evaluated the efficacy of baricitinib in terms of radiographic progression in patients with active RA who were DMARD-naïve or had an inadequate response (IR) to methotrexate (MTX) or to $\geq 1$ conventional synthetic (cs)DMARDs. ${ }^{8-10}$ At weeks 24 and 52, a significant reduction in radiographic progression was observed with the combination of baricitinib $4 \mathrm{mg}$ plus MTX compared with MTX alone in DMARD-naive patients ${ }^{9}$ and with both baricitinib $4 \mathrm{mg}$ and adalimumab on background MTX compared with placebo on background MTX in MTX-IR patients. ${ }^{10}$ Both baricitinib $2 \mathrm{mg}$ and $4 \mathrm{mg}$ were associated with significant inhibition of structural progression compared with placebo on background csDMARDs at 24 weeks in csDMARD-IR patients ${ }^{8}$ and at 1 year for patients who entered into a long-term extension (LTE) study. ${ }^{11}$ As RA requires long-term treatment, the objective of the current analysis was to extend previous findings, assessing radiographic and other efficacy outcomes following up to 2 years of baricitinib treatment for patients completing the originating phase III studies.

\section{METHODS}

\section{Patients}

This analysis included patients who completed RA-BEGIN (DMARD-naïve: NCT01711359), RA-BUILD (csDMARD-IR: NCT01721057) or RA-BEAM (MTX-IR: NCT01710358) and entered into the LTE study RA-BEYOND (NCT01885078). The eligibility criteria for the originating studies have been previously described ${ }^{8-10}$ In brief, eligible patients were $\geq 18$ years old with moderately to severely active RA as defined by the presence of $\geq 6$ tender and $\geq 6$ swollen joints (from a 68/66-joint count) and a high sensitivity C-reactive protein (hsCRP) level $\geq 2 \times$ upper limit of normal (ULN) ( $\geq 6 \mathrm{mg} / \mathrm{L}$ ) for RA-BEAM or hsCRP level $\geq 1.2 \times$ ULN ( $\geq 3.6 \mathrm{mg} / \mathrm{L}$ ) for RA-BEGIN and RA-BUILD. All patients were biologic DMARD-naïve; additionally, patients were csDMARD-naïve or had an insufficient response or were intolerant to MTX or $\geq 1$ csDMARDs.

Patients were eligible for RA-BEYOND if they had completed one of the originating studies. Rescue in the originating study did not preclude entry to RA-BEYOND. Patients were not eligible for participation if they were hypersensitive to baricitinib or if they permanently discontinued baricitinib during the originating study. Patients were also excluded if they had developed any significant medical issues or uncontrolled laboratory abnormalities during the originating study that were, in the opinion of the investigator, an unacceptable risk to the patient.

\section{Study design}

RA-BEYOND is an ongoing (study duration up to 7 years) phase III multicentre LTE of the safety and efficacy of baricitinib in patients who had completed prior baricitinib phase II and phase III clinical trials. The designs of the originating studies have been previously published ${ }^{8-10}$ (see figure 1 and online supplementary methods). Patients receiving blinded baricitinib at the conclusion of an originating study remained on that dose $(2 \mathrm{mg}$ or $4 \mathrm{mg}$, once daily) in RA-BEYOND in a manner blinded to the patient and investigator. In addition, the design of RA-BEYOND included a randomised, blinded substudy on dose step-down from baricitinib $4 \mathrm{mg}$ to $2 \mathrm{mg}$ in patients who had achieved sustained disease control on the higher dose (see online supplementary methods for more detail) ${ }^{12}$

At 52 weeks in the DMARD-naive study (at entry to the LTE), patients receiving MTX or baricitinib $4 \mathrm{mg}$ plus MTX were switched to baricitinib $4 \mathrm{mg}$ monotherapy but could be prescribed MTX or other csDMARDs at the discretion of the investigator at any time point during the LTE. Patients who initiated or increased csDMARDs above the doses used in the randomised, controlled phase of the study were captured through an Interactive Web Response System as rescued. At 52 weeks in the MTX-IR study (at entry to the LTE), patients receiving adalimumab on background MTX were switched to baricitinib $4 \mathrm{mg}$ plus MTX. At 24 weeks in the csDMARD-IR study (at entry to the LTE), patients receiving placebo were switched to baricitinib $4 \mathrm{mg}$ on background csDMARDs. Patients from this study who were rescued from baricitinib $2 \mathrm{mg}$ to $4 \mathrm{mg}$ received baricitinib $4 \mathrm{mg}$ in the LTE; patients who received baricitinib $2 \mathrm{mg}$ in the originating study without rescue continued on $2 \mathrm{mg}$ in the LTE. In the LTE, csDMARDs could be initiated and dosages increased as rescue therapy for patients from the MTX-IR and csDMARD-IR studies who had a Clinical Disease Activity Index score $>10$ at or after 3 months following enrolment into the LTE; patients with normal renal function on baricitinib $2 \mathrm{mg}$ could be rescued to $4 \mathrm{mg}$. Use of biologics was prohibited in the LTE.

\section{Radiographic progression}

Radiographic progression of structural joint damage was determined by the change from baseline in the van der Heijde modified Total Sharp Score $(\mathrm{mTSS})^{13}$ and its subcomponents, the erosion score and joint space narrowing score. In addition, the proportion of patients showing no progression was determined based on change from baseline mTSS ( $\triangle \mathrm{mTSS}$ ) of the originating study, using thresholds of $0,0.5$ or the smallest detectable change (SDC). ${ }^{14}$

To derive the mTSS, radiographs of the hands/wrists and feet were scored, quantifying the extent of bone erosion and joint space narrowing for 44 and 42 joints, respectively, with higher scores representing greater damage. Radiographs were scored by two central readers blinded to chronologic order, patient identity and 


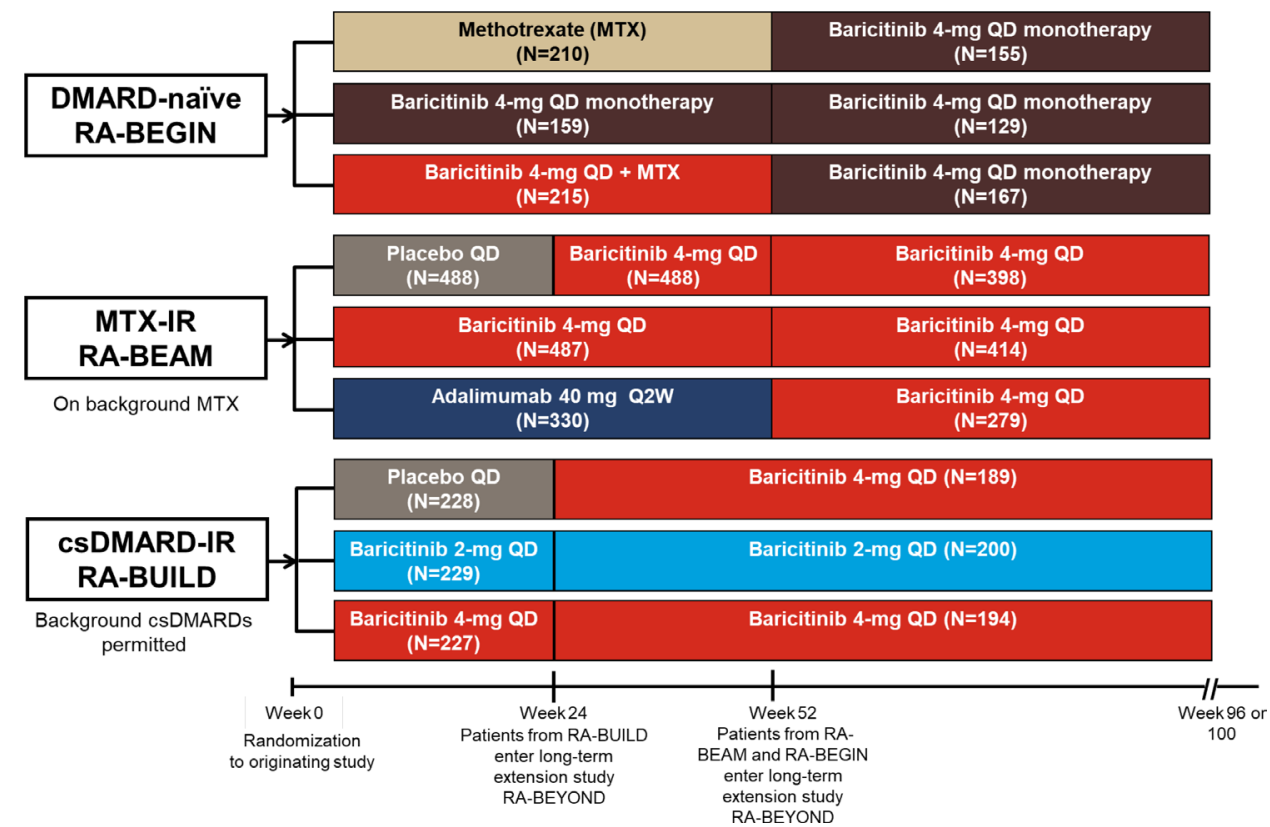

Figure 1 Design for the long-term extension study RA-BEYOND from randomisation in the originating studies. Data are up to 2 years on treatment, corresponding to 100 weeks in RA-BEGIN and RA-BEAM or 96 weeks in RA-BUILD due to the different duration and visit structures of the originating studies. Patients from RA-BEGIN switched to baricitinib 4 mg at entry to RABEYOND at week 52; during RA-BEYOND, MTX or other csDMARDs could be prescribed at any time point at the discretion of the investigators. Patients from RA-BEAM originally randomised to placebo were switched to baricitinib 4 mg at rescue or week 24; patients randomised to adalimumab were switched to baricitinib $4 \mathrm{mg}$ at rescue or entry to RA-BEYOND at week 52. Patients in RA-BUILD initially randomised to placebo switched to baricitinib $4 \mathrm{mg}$ at rescue or week 24 at entry to RABEYOND. Patients who remained on baricitinib 2 mg in RA-BUILD continued on 2 mg in RA-BEYOND; those rescued from baricitinib $2 \mathrm{mg}$ to $4 \mathrm{mg}$ in RA-BUILD received baricitinib $4 \mathrm{mg}$ in RA-BEYOND. Background csDMARDs were permitted in RA-BUILD and RA-BEAM. csDMARD, conventional synthetic DMARD; DMARD, disease-modifying antirheumatic drug; IR, inadequate responder; MTX, methotrexate; N, number of patients randomised and treated; Q2W, once every 2 weeks; QD, once daily; RA, rheumatoid arthritis.

treatment group. The mean score obtained between the two readers was used in the analysis. Baseline initial radiographs taken during the originating study served as the baseline radiographs for comparison throughout the LTE. Results comprise radiographs analysed in the same read campaign that included baseline, 1 year, and either 2-year or early termination time points. Radiographs were taken at study discontinuation if the most recent radiograph was more than 12 weeks earlier.

\section{Clinical efficacy outcomes}

The proportion of patients achieving specific thresholds in clinical efficacy measures was assessed at 12, 24 (DMARD-naïve, MTX-IR and csDMARD-IR studies) and 52 weeks (DMARD-naïve and MTX-IR studies) during the originating studies and every 12 weeks in the LTE up to 2 years. The outcomes examined included a Simplified Disease Activity Index (SDAI) score of $\leq 11$ and a minimal clinically important difference (MCID) of at least 0.22 decrease from baseline on the Health Assessment Questionnaire-Disability Index (HAQ-DI).

\section{Statistical analyses}

Randomised patients who received at least one dose of the assigned study drug were included in the analyses. The analysis population for radiographic progression comprised patients with radiographs at baseline and at least one radiograph from the LTE after the 1 year radiograph. A mixed model for repeated measures (MMRM) was used to analyse continuous variables with treatment, visit and treatment-by-visit-interaction as fixed categorical effects and baseline and baseline-by-visit-interaction as fixed continuous effects. A logistic regression model with treatment included as a factor was used to analyse categorical variables (proportion of patients with no radiographic progression (ie, the proportion of patients meeting one of the thresholds set for $\Delta \mathrm{mTSS} \leq 0, \leq 0.5$ or $\leq \mathrm{SDC}$ ), SDAI, HAQ-DI). The SDC was computed based on week 0 to year 1 and to year 2 changes in mTSS scores assigned by two blinded readers. ${ }^{14}$

Structural data observed after rescue or step-down were included in the analysis, and patients were analysed based on original randomisation. Linear extrapolation $^{15-20}$ was used to impute missing scores at 2 years for patients who discontinued the study early and had radiographs available at 1 year and the early termination visit. The remaining missing scores at 2 years, for which linear extrapolation was not possible due to missing radiographs, were handled by the MMRM analysis (for continuous variables) or by exclusion of patients from the analysis (for the categorical variable of radiographic 
non-progression). The between-group comparisons were assessed with a significance level of 0.05 (two-sided). For SDAI and HAQ-DI data, non-responder imputation was applied after discontinuation of study or study drug. The data cut-off date was September 1, 2016.

\section{RESULTS}

\section{Patient disposition}

Patient disposition is shown in figure 2 according to the treatment groups in the originating studies. Overall, 2573 patients were randomised, and the majority $(\mathrm{N}=2125$; $82.6 \%$ ) entered the LTE. Of these, the requisite radiographic assessments were available for 1893 patients (89.1\%; RA-BEGIN (DMARD-naive): N=410, RA-BEAM (MTX-IR): N=1009, RA-BUILD (csDMARD-IR): N=474) for this analysis. The proportions of patients from RA-BEGIN or RA-BEAM in the analysis population of the LTE completing through 48 weeks (ie, 100 weeks, or 2 years, of treatment; figure 2A,B) and from RA-BUILD completing through 72 weeks (ie, 96 weeks, or 2 years, of treatment; figure 2C) were high $(>94 \%)$ and similar across the original treatment groups in each study. Overall, 13 (3.2\%), $37(3.7 \%)$ and $13(2.7 \%)$ patients from the DMARD-naive, MTX-IR and csDMARD-IR studies, respectively, discontinued from the study during the analysis period in the LTE. In general, there were no major differences in discontinuations across the original treatment arms. The most common reason for discontinuation was an adverse event (DMARD-naive: $n=8$ of 13 discontinuations, $62 \%$; MTX-IR: $\mathrm{n}=20$ of 37 discontinuations, 54.1\%; csDMARD-IR: $\mathrm{n}=6$ of 13 discontinuations, $46.2 \%$ ). For patients discontinuing early, linear extrapolation was applied (when possible) at year 2 for mTSS $(0.0 \%-6.6 \%$ of patients per group; see online supplementary table 1$)$.

The demographic characteristics of the 2-year analysis populations in the LTE were generally similar across the treatment groups at baseline of the originating studies: the majority of patients were white $(>55 \%)$ and female $(>70 \%)$, with a mean age range of 47.5 to 52.9 years (table 1). Clinical characteristics at baseline were similar across the treatment groups of the originating studies, but some characteristics differed across studies due to differences in the eligibility criteria of the originating studies (table 1 ).

\section{Structural progression}

Across the patient populations, treatment with baricitinib was associated with low progression of structural joint damage. For the DMARD-naive patients originating in RA-BEGIN, those on initial baricitinib $4 \mathrm{mg}$ plus MTX showed significantly smaller mean changes from baseline mTSS and erosion scores than patients on initial MTX at both years 1 and 2 ( $\leq \leq 0.001$; figure $3 \mathrm{~A})$; those on initial baricitinib $4 \mathrm{mg}$ monotherapy also had significantly fewer erosions than patients on initial MTX $(p \leq 0.05)$. In agreement with these findings, a greater proportion
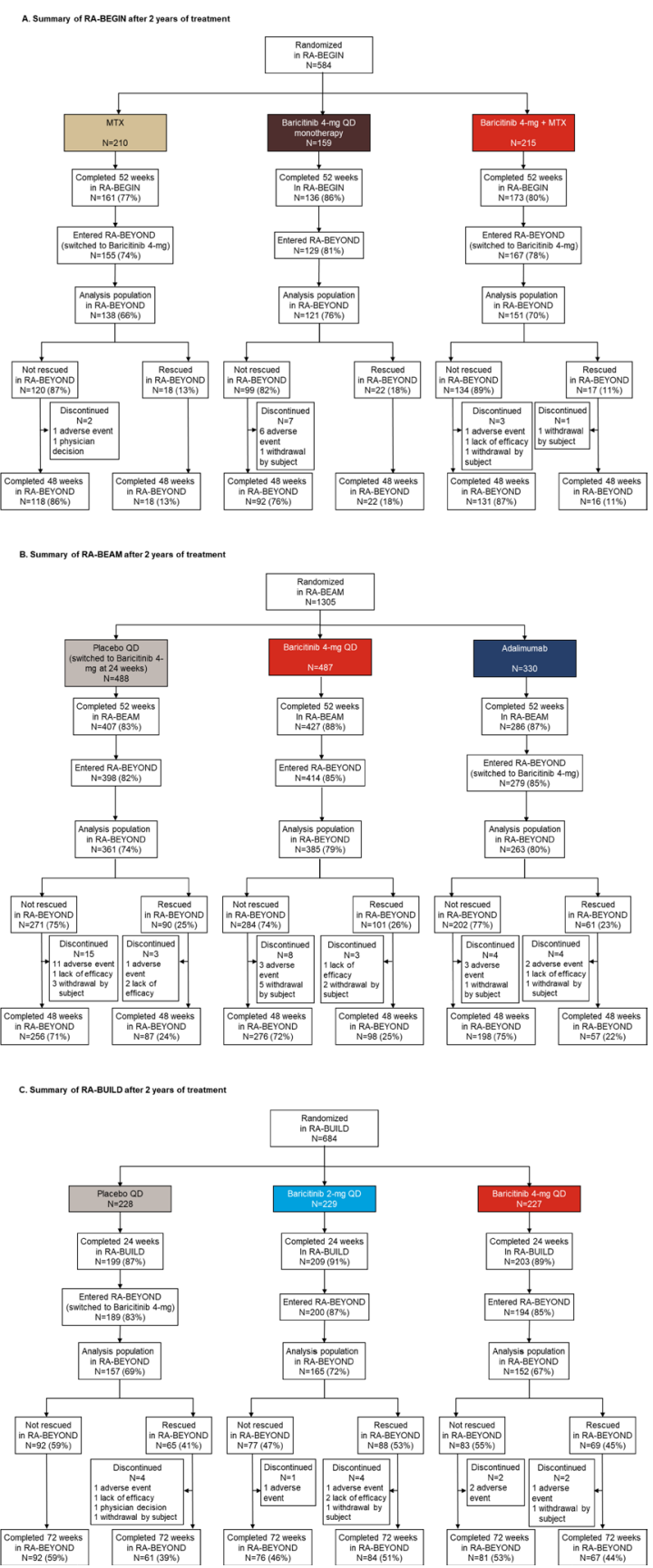

Figure 2 Patient disposition after 2 years of treatment. Summary of the analysis population in RA-BEYOND for patients originally completing (A) RA-BEGIN, (B) RA-BEAM or (C) RA-BUILD. Disposition is shown for up to 2 years on treatment, corresponding to a total of 100 weeks in RA-BEGIN and RA-BEAM (52 weeks in the originating study plus 48 weeks in RA-BEYOND) and 96 weeks in RA-BUILD (24 weeks in originating study plus 72 weeks in RA-BEYOND) due to the different durations and visit structures of the originating studies. Patients on placebo, MTX or adalimumab in the originating studies switched to baricitinib $4 \mathrm{mg}$ at rescue or at 24 or 52 weeks, as detailed in the Study design section. The analysis populations in each group were the patients with radiographic assessments at baseline of the originating study and at least one radiograph from RA-BEYOND after the 1-year radiograph. LTE, longterm extension; MTX, methotrexate; QD, once daily; RA, rheumatoid arthritis. 


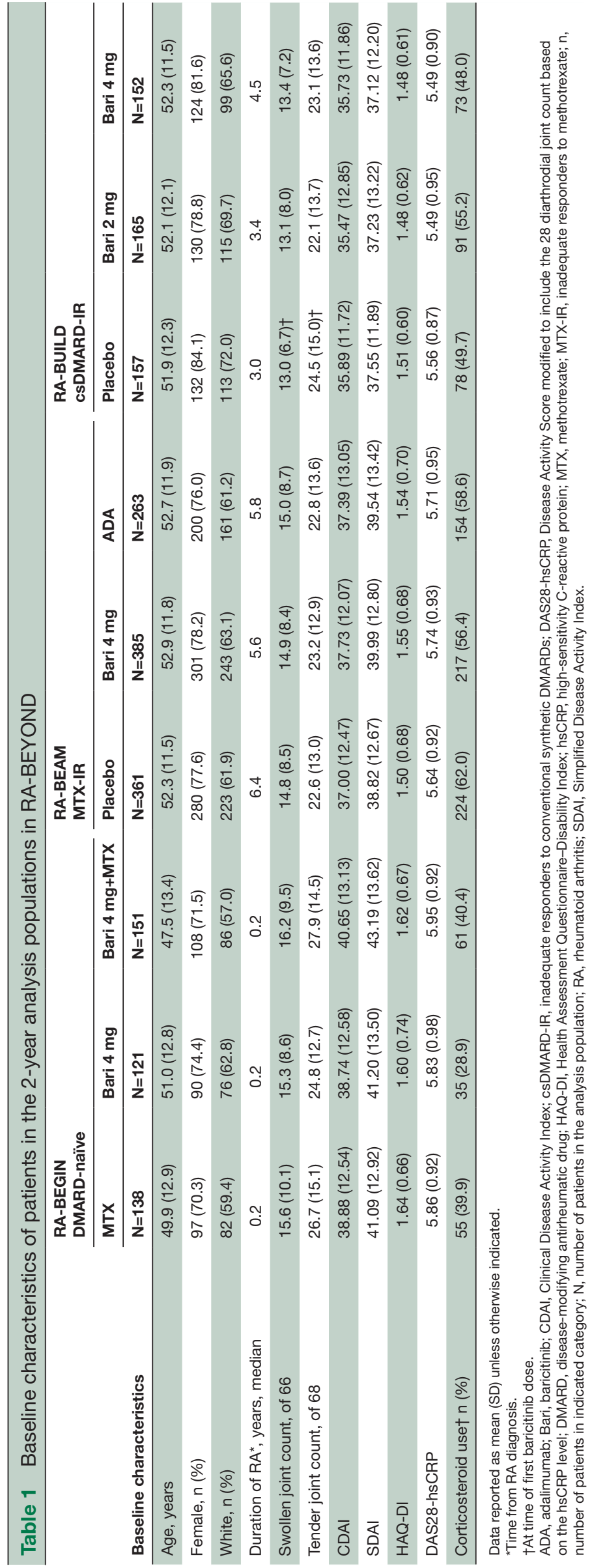




\section{RA-BEGIN}

\section{A. mTSS}

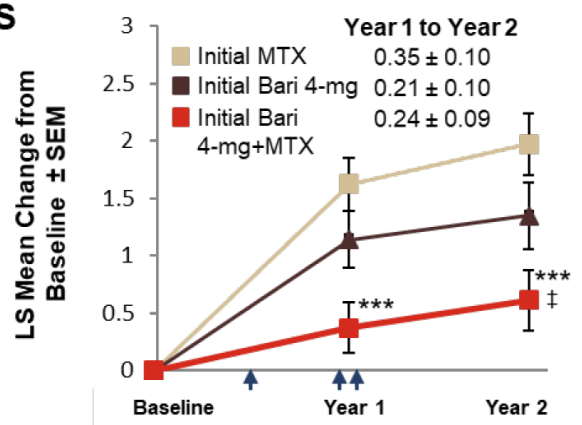

B. ES

C. JSN
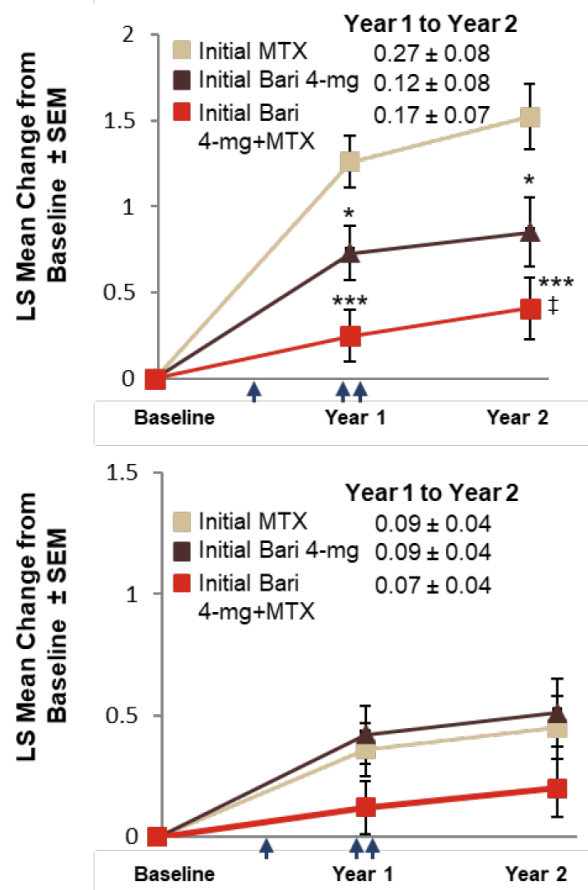

Initial MTX 138

Initial Bari 4-mg 121

Initial Bari 4-mg + MTX 151
136

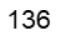

150

\section{RA-BEAM}
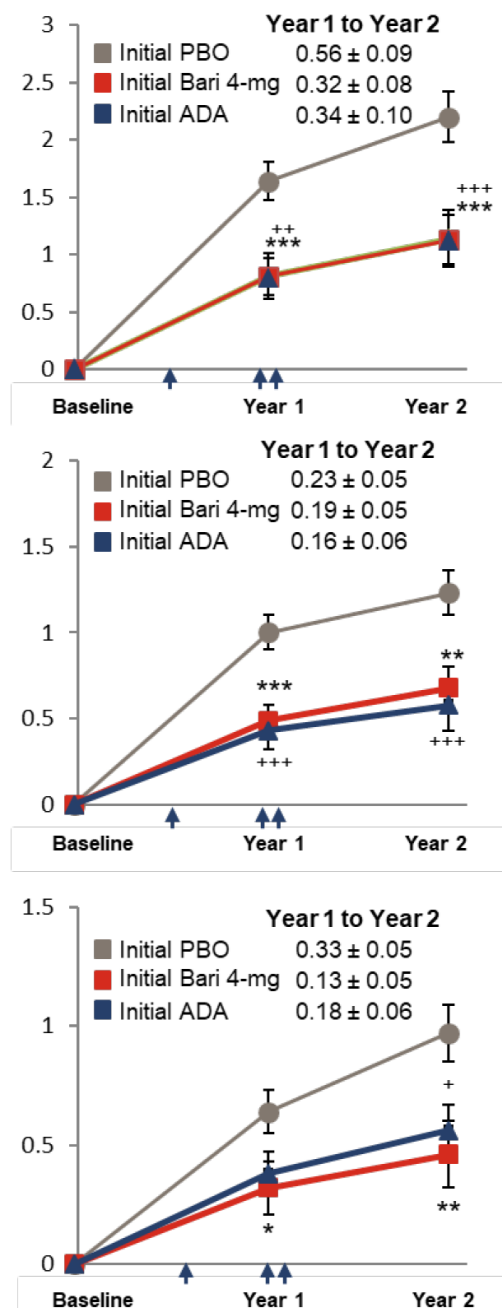

Initial PBO 361
358

380

Initial ADA $263 \quad 260 \quad 260$ Initial Bari 4-mg 152

\section{Year 2 \\ 357}

Initial Bari 2-mg 165
RA-BUILD

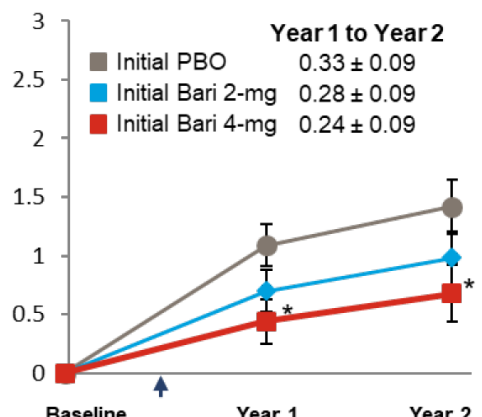

Year 1 to Year 2

$0.19 \pm 0.07$

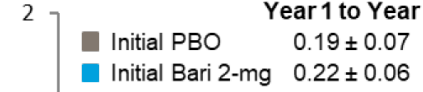

1.5 - Initial Bari 4-mg $0.13 \pm 0.07$
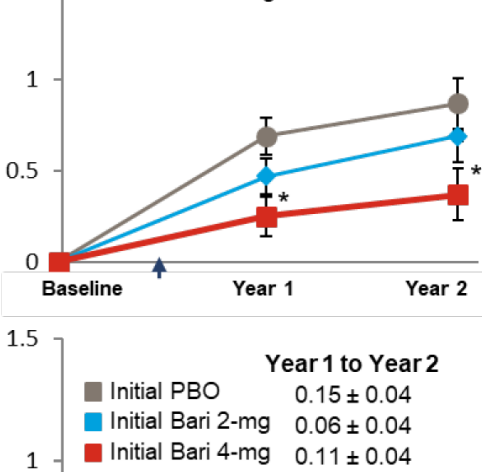

$$
1
$$

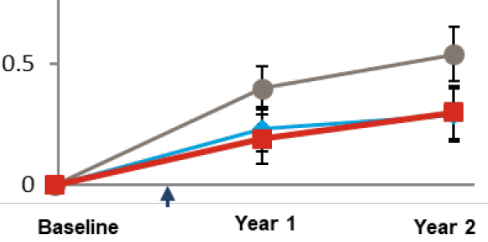

143

157

144 LS mean change from baseline $\left({ }_{ \pm}\right.$SEM) in structural joint damage evaluated using $(A)$ mTSS, (B) ES and (C) JSN for patients in RA-BEYOND originally completing RA-BEGIN, RA-BEAM or RA-BUILD. Time points represent time from randomisation in the originating studies. Indicated treatment groups are according to randomisation in the originating study ('initial' denotes initial randomised treatment group). Patients on PBO, MTX or ADA in the originating studies switched to baricitinib 4 mg at rescue or at 24 weeks (PBO; single arrow) or 52 weeks (MTX and ADA; double arrow) as detailed in the Study design section. Tables represent the number of patients for whom data were available for each time point and study. Comparisons were analysed using a mixed model for repeated measures. Missing scores at 2 years were imputed using linear extrapolation based on data collected between 1 and 2 years. SE of the mean ${ }^{*} \mathrm{p} \leq 0.05,{ }^{* *} \mathrm{p} \leq 0.01,{ }^{* * *} \mathrm{p} \leq 0.001$ baricitinib 4 mg vs PBO (RA-BEAM; RA-BUILD) or MTX (RA-BEGIN); ${ }^{+} \mathrm{p} \leq 0.05,{ }^{++} \mathrm{p} \leq 0.01,{ }^{++} \mathrm{p} \leq 0.001$ ADA vs PBO (RA-BEAM); $\neq \mathrm{p} \leq 0.05$ baricitinib 4 mg vs baricitinib $4 \mathrm{mg}$ plus MTX (RA-BEGIN). ADA, adalimumab; Bari, baricitinib; ES, erosion score; JSN, joint space narrowing; LS, least squares; mTSS, modified Total Sharp Score; MTX, methotrexate; PBO, placebo; RA, rheumatoid arthritis.

of patients who had received initial baricitinib therapies in the DMARD-naïve study had no radiographic progression compared with patients who had received initial MTX monotherapy. These differences reached statistical significance at both years 1 and 2 for the initial baricitinib $4 \mathrm{mg}$ plus MTX group when any of the thresholds were used (figure 4; see online supplementary table 2) and for the baricitinib $4 \mathrm{mg}$ monotherapy group when either the $\Delta$ mTSS $\leq 0$ or $\Delta$ mTSS $\leq 0.5$ thresholds were used (see online supplementary table 2). For MTX-IR patients originating in RA-BEAM, those on initial baricitinib $4 \mathrm{mg}$ had statistically significantly lower radiographic progression at years 1 and 2 than patients on initial placebo, and similar progression to patients on initial adalimumab (figure 3B). Both the baricitinib 4 $\mathrm{mg}$ and initial adalimumab groups also showed similar proportions of patients with mTSS nonprogression using any of the thresholds, which were statistically significantly 
Year 1

Year 2

\section{A. RA-BEGIN}
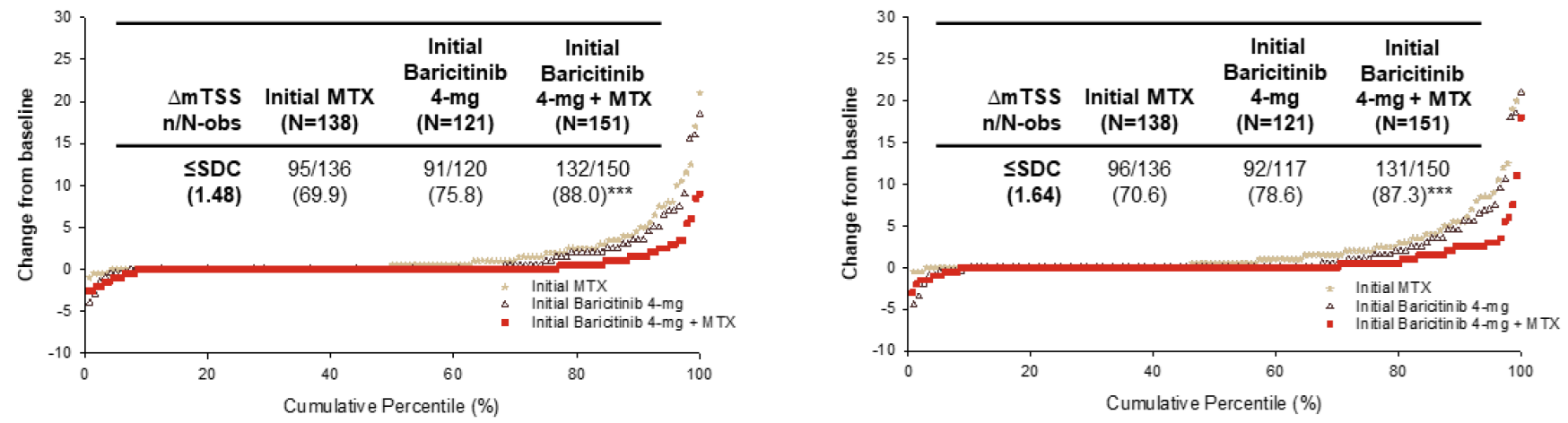

B. RA-BEAM
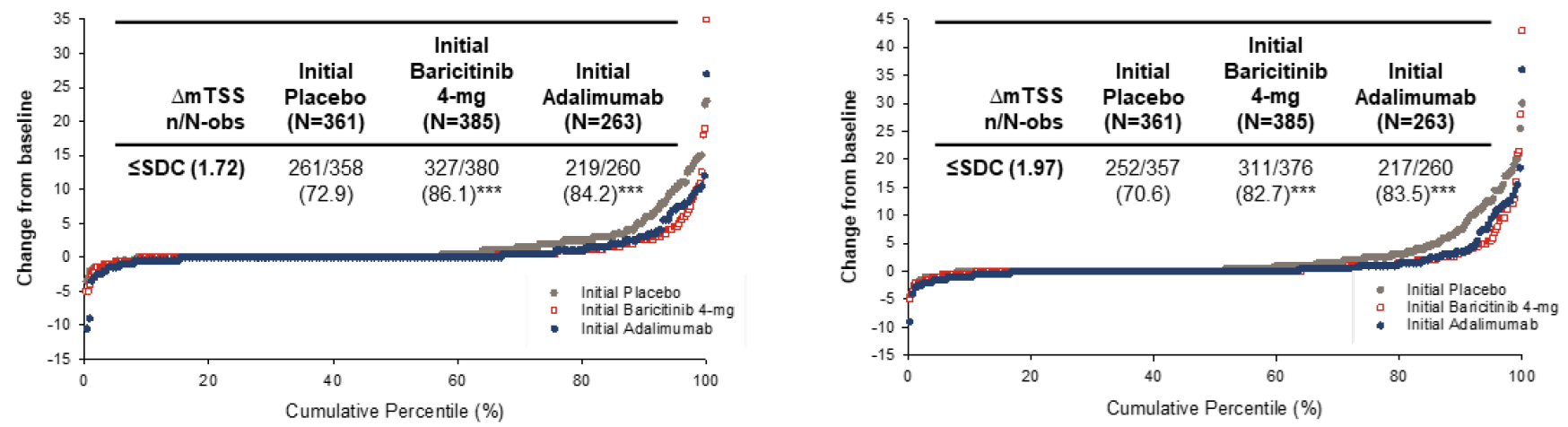

C. RA-BUILD
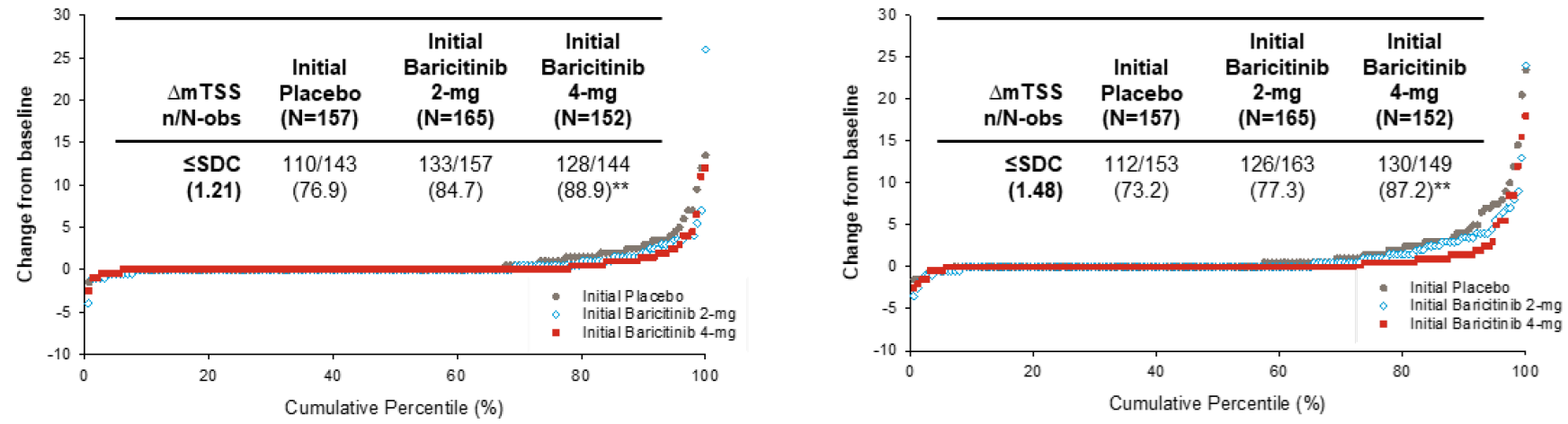

Figure 4 Patient-level radiographic progression of structural joint damage at year 1 and year 2 by original randomisation. Panels show radiographic progression in structural joint damage evaluated using cumulative percentile change in the modified Total Sharp Score (mTSS) from baseline at year 1 and year 2 for patients in RA-BEYOND originally completing (A) RA-BEGIN, (B) RA-BEAM or (C) RA-BUILD. Each point represents an individual patient. Indicated treatment groups are according to randomisation in the originating study ('initial' denotes initial randomised treatment group). Inserted tables show $\mathrm{n}$ and percentage non-progression as defined by $\leq$ SDC. Patients on placebo, MTX or adalimumab in the originating studies switched to baricitinib $4 \mathrm{mg}$ at rescue or at 24 or 52 weeks, as detailed in the Study design section. ${ }^{\star \star} p \leq 0.01,{ }^{* \star \star} p \leq 0.001$ vs placebo (RA-BEAM; RA-BUILD) or MTX (RA-BEGIN). $\triangle$, change from baseline; mTSS, modified Total Sharp Score; MTX, methotrexate; $\mathrm{n}$, number of patients meeting threshold; $\mathrm{N}$, number of patients with non-missing baseline and $\geq 1$ non-missing post-baseline mTSS data; N-obs, number of patients included in analysis; RA, rheumatoid arthritis; SDC, smallest detectable change.

higher than the proportion of patients showing no radiographic progression in the initial placebo group at both years 1 and 2 (figure 4; online supplementary table 2). For csDMARD-IR patients who had completed RA-BUILD, those on initial baricitinib $4 \mathrm{mg}$ showed significantly smaller mean changes in mTSS and erosions $(\mathrm{p} \leq 0.05$; figure $2 \mathrm{C}$ ) and a greater proportion showed no radiographic progression (figure 4; online supplementary table 2) compared with patients on initial placebo at years 1 and 2 ( $p \leq 0.05$; figure 2C). Progression with initial baricitinib $2 \mathrm{mg}$ was numerically lower than with initial placebo (figure $3 \mathrm{C}$ ); the proportion of patients showing 
no mTSS progression was also higher in the baricitinib $2 \mathrm{mg}$ group compared with the placebo group at both 1 and 2 years, but these differences did not reach statistical significance.

For all treatment groups, lower rates of change in mTSS were observed between years 1 and 2 (when all patients were on baricitinib therapies) than between baseline and year 1 (figure 3). Patients originally randomised to MTX or placebo exhibited greater increases in mTSS between years 1 and $2(0.35 \pm 0.10$ in RA-BEGIN; $0.56 \pm 0.09$ in RA-BEAM; $0.33 \pm 0.09$ in RA-BUILD) than those originally randomised to baricitinib treatment groups $(0.21 \pm 0.01$ and $0.24 \pm 0.09$ for baricitinib $4 \mathrm{mg}$ and baricitinib $4 \mathrm{mg}$ plus MTX, respectively, in RA-BEGIN; $0.32 \pm 0.08$ for baricitinib $4 \mathrm{mg}$ in RA-BEAM; $0.28 \pm 0.09$ and $0.24 \pm 0.09$ for 2 and $4 \mathrm{mg}$, respectively, in RA-BUILD) (figure 3). Individual patient-level changes in radiographic progression from baseline were in accordance with these data at years 1 and 2 (figure 4 ).

\section{Clinical efficacy}

The majority $(>60 \%)$ of patients across studies receiving baricitinib treatment achieved SDAI $\leq 11$ at entry into the LTE study RA-BEYOND (figure 5A), which was sustained for up to 2 years of treatment. Similarly, the majority of patients on baricitinib $4 \mathrm{mg}$ across the studies had functional benefit from treatment, with approximately $75 \%$ of patients on baricitinib who met or exceeded the HAQ-DI MCID $(\geq 0.22)$ by week 12 in the originating studies (figure $5 \mathrm{~B}$ ). More than $60 \%$ of patients across studies met or exceeded the HAQ-DI MCID $(\geq 0.22)$ at both years 1 and 2 . The patients receiving the $2 \mathrm{mg}$ dose through 2 years showed similar results to those receiving the $4 \mathrm{mg}$ dose in terms of both SDAI $\leq 11$ and HAQ-DI MCID ( $\geq 0.22)$ response.

\section{DISCUSSION}

The current analysis is a report on radiographic outcomes in the LTE study, RA-BEYOND, including

\section{A. SDAI $\leq 11$}

\section{RA-BEGIN}

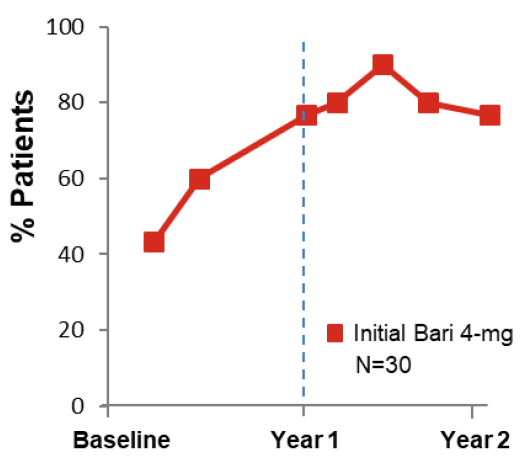

B. HAQ-DI $\geq 0.22$ (MCID)

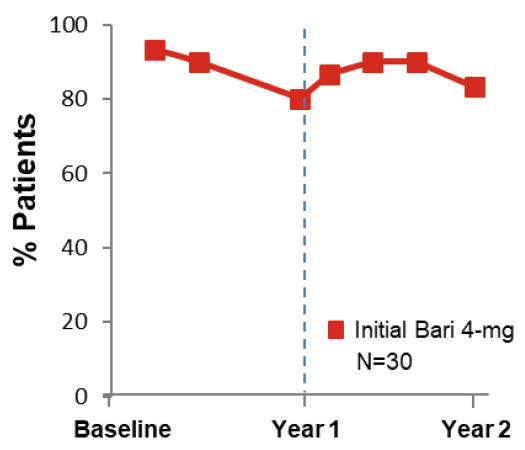

RA-BEAM
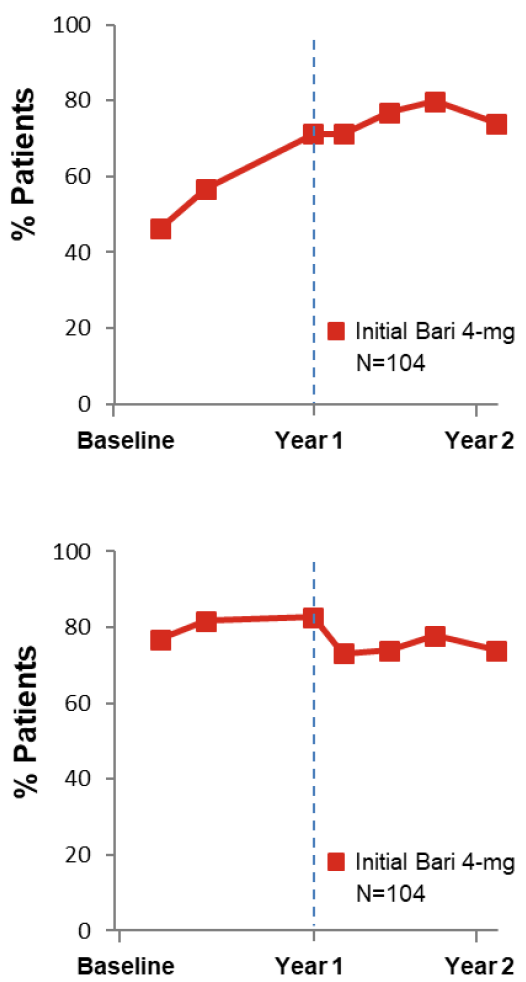

RA-BUILD

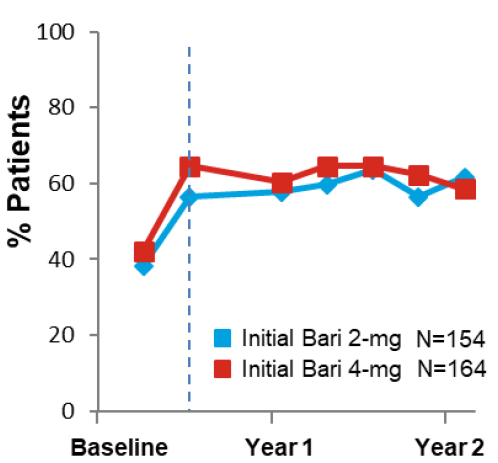

Figure 5 Proportion of patients (NRI) on baricitinib achieving SDAI $\leq 11$ and meeting or exceeding HAQ-DI MCID ( $\geq 0.22)$ up to year 2 by original randomisation groups. Baseline in the originating study was used in the calculation of response. Time points represent time from randomisation in originating study, with year 1 and year 2 corresponding respectively to week 52 and week 100 for RA-BEGIN and RA-BEAM and to week 48 and week 96 for RA-BUILD due to the different durations and visit structures of the originating studies. Time points between year 1 and year 2 are in 12-week intervals. Dotted lines indicate entry into the long-term extension study. Analyses exclude patients who were rescued in the originating studies. NRI was applied without regard to rescue status in RA-BEYOND. Data after patients stepped down to baricitinib 2 mg were imputed using the response rate of the patients who continued with baricitinib $4 \mathrm{mg}$. 'Initial' denotes initial randomised treatment group. Bari, baricitinib; HAQ-DI, Health Assessment Questionnaire-Disability Index; MCID, minimally clinically important difference; N, number of patients not rescued in the originating study, who continued on the same dose in RA-BEYOND and entered RA-BEYOND 96 weeks before the cut-off date; NRI, non-responder imputation; SDAI, Simplified Disease Activity Index; RA, rheumatoid arthritis. 
patients from three studies with active RA who were either DMARD-naive or had an inadequate response to MTX or $\geq 1$ csDMARDs. The key finding is that baricitinib inhibited structural progression for up to 2 years of treatment, as assessed by total mTSS, erosions and joint space narrowing. Patients initially started on MTX or placebo exhibited significantly larger changes from baseline mTSS than those started on baricitinib $4 \mathrm{mg}$, either alone or in combination with MTX, with a higher proportion of patients on initial baricitinib therapies exhibiting non-progression at years 1 and 2. Further, patients on initial baricitinib therapies exhibited minimal changes in mTSS between years 1 and 2 compared with those on initial placebo or MTX, indicating that the inhibition of structural progression associated with initial baricitinib persisted during the second year of treatment. In MTX-IR patients completing RA-BEAM, radiographic outcomes at 1 year were similar between patients initially randomised to baricitinib $4 \mathrm{mg}$ and adalimumab and remained similar at 2 years (ie, 1 year following the switch of initial adalimumab-treated patients to baricitinib $4 \mathrm{mg}$ ). These data are consistent with, and further extend, the original findings of the three studies, which reported significant reductions in radiographic progression in the baricitinib treatment groups at the end of the randomised study periods ${ }^{8-10}$ and at 1 year for the patients who had completed the csDMARD-IR study RA-BUILD and entered the LTE. ${ }^{11}$ Collectively, the data show that baricitinib, as a monotherapy or in combination with MTX/csDMARDs, provides sustained structural benefits in many patients with active RA, including those who have previously failed to adequately improve on MTX or other csDMARDs. Further, the data indicate that patients originally randomised to placebo or MTX and then switched to baricitinib $4 \mathrm{mg}$ at 24 weeks continue to exhibit worse structural progression than patients originally randomised to baricitinib, even after 2 years of treatment, indicating that early intervention with baricitinib therapies is beneficial in the long term.

For the current analysis, radiographs at baseline and 1 year were reread during scoring of the year 2 radiographs. Consistent with previous studies which showed statistically significant structural benefit of baricitinib 2 $\mathrm{mg}$ and $4 \mathrm{mg}$ at 24 and 52 weeks, ${ }^{811}$ the current study demonstrates significant benefit with initial baricitinib 4 $\mathrm{mg}$ in this population with up to 2 years of treatment. In addition, numerically greater reductions in the mean change from baseline mTSS and a higher proportion of patients showing no mTSS progression were observed in patients treated with baricitinib $2 \mathrm{mg}$ compared with those on initial placebo who, at years 1 and 2, would have been treated with baricitinib $4 \mathrm{mg}$ for 24 and 72 weeks, respectively. Collectively, the data indicate that both doses are effective at reducing structural progression with the best outcomes observed when treatment was started sooner and a more robust structural benefit achieved with baricitinib $4 \mathrm{mg}$.
In keeping with the radiographic progression findings, the majority of patients across the three originating studies receiving baricitinib therapies achieved SDAI $\leq 11$ and HAQ-DI MCID by entry to the LTE, and this was maintained for up to 2 years on treatment. These data suggest that the sustained improvements in disease activity observed with long-term baricitinib treatment were associated with structural benefit and improved function. Safety was not examined in the current report as separate assessments of the safety of long-term baricitinib treatment have been conducted recently, which reported no significant new safety signals during the LTE up to 288 weeks. ${ }^{11} 2122$ Notably, a recent comprehensive safety report has been published, which used the same data cut-off date as the current paper and large integrated data analysis sets from the baricitinib clinical programme. $^{22}$ Across anaysis sets, infections were the most common adverse events reported in the baricitinib groups, but no notable differences were observed across treatment groups in the rates of serious adverse events including deaths, permanent discontinuations due to adverse events, malignancies, major adverse cardiovascular events and serious infections. ${ }^{22}$ Collectively, the data indicate that baricitinib treatment has a long-term clinical benefit with an acceptable safety profile.

There are some limitations to this analysis. Although this study included a large patient population, representing both DMARD-naïve and csDMARD-IR patients with active RA, this analysis included patients with varying treatment regimens and exposures to baricitinib due to differences in the designs of the three originating studies and to rescue and taper during the LTE. The study design did not permit comparison between active treatments and doses across studies. In addition, the LTE was open label, and patients were aware that they were receiving baricitinib. However, both patient and investigator were blinded to original randomised treatment assignments and the dose of baricitinib. Further, all radiographs were scored by two central readers who were blinded to all clinical information.

In conclusion, treatment with once-daily baricitinib was associated with low rates of radiographic progression for up to 2 years of treatment. Patients on initial baricitinib showed significantly less progression than those starting with placebo or MTX then switched to baricitinib, and comparable progression with those starting with adalimumab then switched to baricitinib. These differences in the level of progression were observed at both years 1 and 2, indicating a persistent structural benefit with longterm baricitinib treatment. For csDMARD-IR patients, treatment with baricitinib $2 \mathrm{mg}$ and $4 \mathrm{mg}$ was associated with reduced radiographic progression compared with initial placebo, with baricitinib $4 \mathrm{mg}$ providing more robust evidence than the $2 \mathrm{mg}$ dose.

\section{Author affiliations}

${ }^{1}$ Rheumatology, Leiden University Medical Center, Leiden, The Netherlands

${ }^{2}$ Rheumatology, Diakonhjemmet Hospital, Oslo, Norway 
${ }^{3}$ Rheumatology, University of Colorado, Englewood, Colorado, USA

${ }^{4}$ First Department of Internal Medicine, University of Occupational and Environmental Health, Kitakyushu, Japan

${ }^{5}$ Eli Lilly and Company, Indianapolis, Indiana, USA

${ }^{6}$ Leeds Institute of Rheumatic and Musculoskeletal Medicine, Leeds, UK ${ }^{7}$ Leeds Teaching Hospitals NHS Trust, NIHR Leeds Musculoskeletal Biomedical Research Unit, Leeds, UK

Acknowledgements We thank the patients, their families and the study personnel who participated in these clinical trials. These data were first presented at the Annual European Congress of Rheumatology 2017 (EULAR Abstract FRI0087; conference paper in Annals of the Rheumatic Diseases 76(Suppl 2):510.2-511). The studies were sponsored by Eli Lilly and Company (Indianapolis, Indiana, USA) and Incyte Corporation. Eli Lilly and Company sponsored the production of this manuscript. Syneos Health provided medical writing (Kaye L Stenvers) and editing (Antonia Baldo and Rod Everhart) support.

Contributors DvdH, PE and MS designed the experiments and analysed and interpreted the data; $\mathrm{LX}$ and RAO analysed and interpreted the data; GM, MC, TI and YT interpreted the data. All authors assisted in drafting and/or revising the manuscript, contributed to its intellectual content and approved the final version to be submitted. The authors take full responsibility for the manuscript.

Funding The studies were sponsored by Eli Lilly and Company (Indianapolis, Indiana, USA) and Incyte Corporation. Eli Lilly and Company sponsored the production of this manuscript.

Competing interests DvdH: received consulting fees from AbbVie, Amgen, Astellas, AstraZeneca, BMS, Boehringer Ingelheim, Celgene, Daiichi, Eli-Lilly, Galapagos, Gilead, Janssen, Merck, Novartis, Pfizer, Regeneron, Roche, Sanofi, UCB and is director of Imaging Rheumatology bv. MS: received consulting and/or speaking fees from AbbVie, Bristol-Myers Squibb, Eli Lilly and Company, Johnson \& Johnson, UCB. YT: received speaking fees and/or honoraria from Daiichi-Sankyo, Astellas, Eli Lilly and Company, Chugai, Sanofi, Abbvie, Pfizer, YL Biologics, Bristol-Myers, Glaxo-SmithKline, UCB, Mitsubishi-Tanabe, Novartis, Eisai, Takeda, Janssen, Asahi-kasei and has received research grants from Mitsubishi-Tanabe, Bristol-Myers, Eisai, Chugai, Takeda, Abbvie, Astellas, Daiichi-Sankyo, Ono, MSD, Taisho-Toyama. LX, GM, TI, MC, RAO: employees and stock owners of Eli Lilly and Company. PE: received grant/research support or consulting support from Abbott, AbbVie, Bristol Myers Squibb, Eli Lilly and Company, Gilead, MSD, Novartis, Pfizer, Roche, Samsung, Takeda, UCB.

Patient consent for publication Obtained.

Ethics approval All studies were conducted with the approval of an institutional review board and in accordance with the ethical principles of the Declaration of Helsinki of 1975 (revised in 1983) and the Good Clinical Practice guidelines.

Provenance and peer review Not commissioned; externally peer reviewed.

Data availability statement Data are available on reasonable request. Lilly provides access to the individual patient data from studies on approved medicines and indications as defined by the sponsor-specific information (available at https:// clinicalstudydatarequest.com). This access is provided in a timely fashion after the primary publication is accepted. Researchers need to have an approved research proposal submitted online (https://clinicalstudydatarequest.com). Access to the data will be provided in a secure data sharing environment after signing a data sharing agreement.

Open access This is an open access article distributed in accordance with the Creative Commons Attribution Non Commercial (CC BY-NC 4.0) license, which permits others to distribute, remix, adapt, build upon this work non-commercially, and license their derivative works on different terms, provided the original work is properly cited, appropriate credit is given, any changes made indicated, and the use is non-commercial. See: http://creativecommons.org/licenses/by-nc/4.0/.

\section{REFERENCES}

1. Colmegna I, Ohata BR, Menard HA. Current understanding of rheumatoid arthritis therapy. Clin Pharmacol Ther 2012;91:607-20.

2. Dadoun S, Zeboulon-Ktorza N, Combescure C, et al. Mortality in rheumatoid arthritis over the last fifty years: systematic review and meta-analysis. Joint Bone Spine 2013;80:29-33.
3. Combe B, Landewe R, Daien $\mathrm{Cl}$, et al. Update of the EULAR recommendations for the management of early arthritis. Ann Rheum Dis 2016;2017:948-59.

4. Singh JA, Saag KG, Bridges SL, et al. 2015 American College of Rheumatology guideline for the treatment of rheumatoid arthritis. Arthritis Rheumatol 2016;68:1-26.

5. Smolen JS, Landewé R, Bijlsma J, et al. EULAR recommendations for the management of rheumatoid arthritis with synthetic and biological disease-modifying antirheumatic drugs: 2016 update. Ann Rheum Dis 2017;76:960-77.

6. Fridman JS, Scherle PA, Collins R, et al. Selective inhibition of JAK1 and Jak2 is efficacious in rodent models of arthritis: preclinical characterization of INCB028050. J Immunol 2010;184:5298-307.

7. O'Shea JJ, Holland SM, Staudt LM. JAKs and STATs in immunity, immunodeficiency, and cancer. N Engl J Med 2013;368:161-70.

8. Dougados M, van der Heijde D, Chen Y-C, et al. Baricitinib in patients with inadequate response or intolerance to conventional synthetic DMARDs: results from the RA-BUILD study. Ann Rheum Dis 2017;76:88-95.

9. Fleischmann R, Schiff M, van der Heijde D, et al. Baricitinib, methotrexate, or combination in patients with rheumatoid arthritis and no or limited prior disease-modifying antirheumatic drug treatment. Arthritis Rheumatol 2017;69:506-17.

10. Taylor PC, Keystone EC, van der Heijde D, et al. Baricitinib versus placebo or adalimumab in rheumatoid arthritis. N Engl J Med 2017;376:652-62.

11. van der Heijde D, Dougados M, Chen Y-C, et al. Effects of baricitinib on radiographic progression of structural joint damage at 1 year in patients with rheumatoid arthritis and an inadequate response to conventional synthetic disease-modifying antirheumatic drugs. RMD Open 2018:4:e000662.

12. Takeuchi T, Genovese MC, Haraoui B, et al. Dose reduction of baricitinib in patients with rheumatoid arthritis achieving sustained disease control: results of a prospective study. Ann Rheum Dis 2018.

13. van der Heijde D. How to read radiographs according to the Sharp/ van der Heijde method. J Rheumatol 2000;27:261-3.

14. Bruynesteyn Ket al. Deciding on progression of joint damage in paired films of individual patients: smallest detectable difference or change. Ann Rheum Dis 2005;64:179-82.

15. Cohen SB, Emery P, Greenwald MW, et al. Rituximab for rheumatoid arthritis refractory to anti-tumor necrosis factor therapy: results of a multicenter, randomized, double-blind, placebo-controlled, phase III trial evaluating primary efficacy and safety at twenty-four weeks. Arthritis Rheum 2006;54:2793-806.

16. Keystone E, Heijde DVD, Mason D, et al. Certolizumab pegol plus methotrexate is significantly more effective than placebo plus methotrexate in active rheumatoid arthritis: findings of a fifty-twoweek, phase III, multicenter, randomized, double-blind, placebocontrolled, parallel-group study. Arthritis Rheum 2008;58:3319-29.

17. , Emery P, Peterfy CG, et al. Rituximab inhibits structural joint damage in patients with rheumatoid arthritis with an inadequate response to tumour necrosis factor inhibitor therapies. Ann Rheum Dis 2009;68:216-21.

18. Keystone EC, Kavanaugh AF, Sharp JT, et al. Radiographic, clinical, and functional outcomes of treatment with adalimumab (a human anti-tumor necrosis factor monoclonal antibody) in patients with active rheumatoid arthritis receiving concomitant methotrexate therapy: a randomized, placebo-controlled, 52-week trial. Arthritis Rheum 2004;50:1400-11.

19. Smolen J, Landewe RB, Mease $P$, et al. Efficacy and safety of certolizumab pegol plus methotrexate in active rheumatoid arthritis: the RAPID 2 study. A randomised controlled trial. Ann Rheum Dis 2009;68:797-804.

20. Smolen JS, Beaulieu A, Rubbert-Roth A, et al. Effect of interleukin-6 receptor inhibition with tocilizumab in patients with rheumatoid arthritis (OPTION study): a double-blind, placebo-controlled, randomised trial. Lancet 2008;371:987-97.

21. Keystone EC, Genovese MC, Schlichting DE, et al. Safety and efficacy of baricitinib through 128 weeks in an open-label, longterm extension study in patients with rheumatoid arthritis. J Rheumatol 2018;45:14-21.

22. Smolen JS, Genovese MC, Takeuchi T, et al. Safety profile of baricitinib in patients with active rheumatoid arthritis with over 2 years median time in treatment. $J$ Rheumatol 2019;46:7-18. 


\section{Correction: Low rates of radiographic progression of structural joint damage over 2 years of baricitinib treatment in patients with rheumatoid arthritis}

van der Heijde D, Schiff M, Tanaka Y, et al. Low rates of radiographic progression of structural joint damage over 2 years of baricitinib treatment in patients with rheumatoid arthritis. RMD Open 2019;5:e000898. doi: 10.1136/rmdopen-2019-000898

The authors want to alert readers to the following errors in regard to Figure 3.

1. In panels A (mTSS) and B (ES) for the RA-BEGIN graphs, the symbol ( $\$$ ) indicating the significance level for the comparison between the baricitinib 4-mg and baricitinib 4-mg plus MTX treatment groups (denoting $\mathrm{p} \leq 0.05$ ) should be over the 1 year time point, not the 2 year time point in both graphs.

2. In panel A (mTSS) for RA-BEAM, for the adalimumab versus placebo comparison at 2 years, the significance should have been shown as $++(\mathrm{p} \leq 0.01)$ and not +++ $(\mathrm{p} \leq 0.001)$.

3. In the Results text, p.8, line 12 (relating to Figure 3, panel A) '...baricitinib treatment groups $(0.21 \pm 0.01 \ldots$ ' should read: '...baricitinib treatment groups $(0.21 \pm 0.10 \ldots$ '

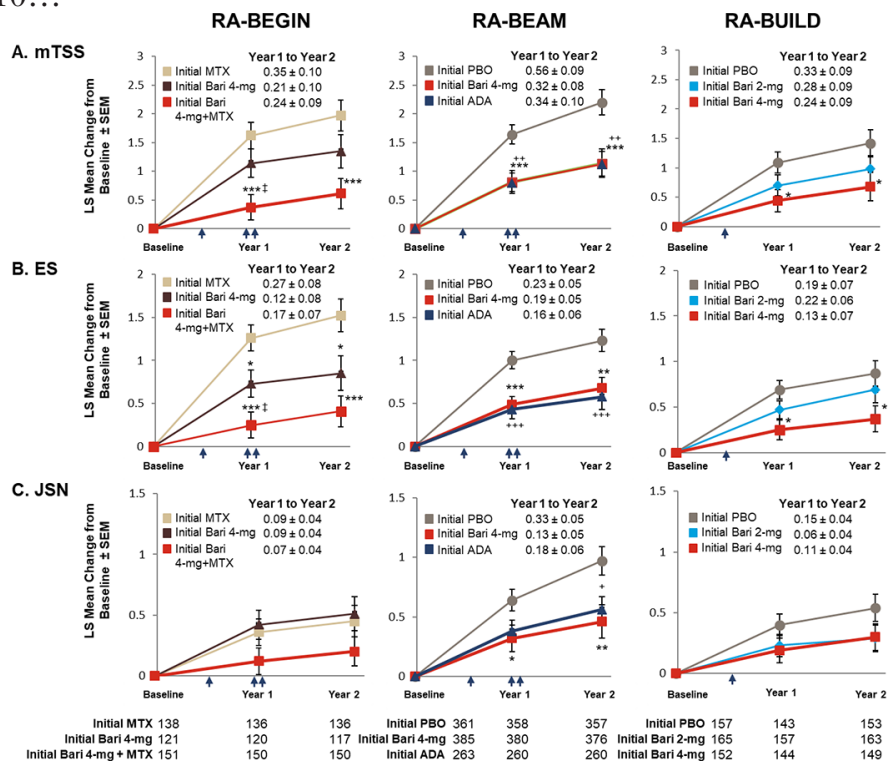

Figure 3 Inhibition of radiographic progression of structural joint damage at year 1 and year 2 by original randomization. The LS mean change from baseline $( \pm S E M)$ in structural joint damage evaluated using (A) mTSS, (B) ES, and (C) JSN for patients in RA-BEYOND originally completing RA-BEGIN, RA-BEAM, or RA-BUILD. Time points represent time from randomization in the originating studies. Indicated treatment groups are according to randomization in the originating study ('initial' denotes initial randomized treatment group). Patients on PBO, MTX, or ADA in the originating studies switched to baricitinib $4 \mathrm{mg}$ at rescue or at 24 weeks (PBO; single arrow) or 52 weeks (MTX and ADA; double arrow) as detailed in the study design. Tables represent the number of patients for whom data were available for each time point and study. Comparisons were analysed using a mixed model for repeated measures. Missing scores at 2 years were imputed using linear extrapolation based on data collected between 1 and 2 years. ADA, adalimumab; Bari, baricitinib; ES, erosion score; JSN, joint space narrowing; LS, least squares; mTSS, modified total Sharp score; MTX, methotrexate; PBO, placebo; RA, rheumatoid arthritis; SEM, SE of the mean ${ }^{*} \mathrm{p} \leq 0.05,{ }^{* *} \mathrm{p} \leq 0.01,{ }^{* *} \mathrm{p} \leq 0.001$ baricitinib $4 \mathrm{mg}$ vs PBO (RA-BEAM; RA-BUILD) or MTX (RABEGIN); ${ }^{+} \leq 0.05,{ }^{++} p \leq 0.01,{ }^{++} p \leq 0.001$ ADA vs PBO (RA-BEAM); $\ddagger \mathrm{p} \leq 0.05$ baricitinib $4 \mathrm{mg}$ vs baricitinib $4 \mathrm{mg}$ plus MTX (RA-BEGIN).

Open access This is an open access article distributed in accordance with the Creative Commons Attribution Non Commercial (CC BY-NC 4.0) license, which permits others to distribute, remix, adapt, build upon this work non-commercially, and license their derivative works on different terms, provided the original work is properly cited, appropriate credit is given, any changes made indicated, and the use is non-commercial. See: http://creativecommons. org/ licenses/by-nc/4.0/ 
C Author(s) (or their employer(s)) 2019. Re-use permitted under CC BY-NC. No commercial re-use. See rights and permissions. Published by BMJ.

RMD Open 2019;5. doi:10.1136/rmdopen-2019-000898corr1

(D) Check for updates 